\title{
Comportamento Eletroquímico da Liga Ti-12mo-13nb envelhecida para aplicação Biomédica
}

\section{Electrochemical corrosion behavior in aged Ti-12mo-13nb aged to Biomedical Applications}

\author{
Tales Vieira Silva ${ }^{1}$ \\ Iranildes dos Santos ${ }^{2}$ \\ Sinara Borborema Gabriel ${ }^{2}$
}

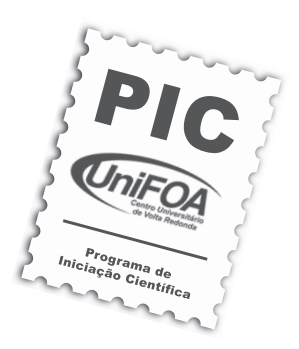

Artigo

Original

Original

Paper

\begin{abstract}
Resumo
Palavras-chave:

Biomateriais

Ligas de Titânio

Corrosão

Microestrutura

Consideráveis esforços têm sido feitos para explorar novas ligas de Ti visando aplicações ortopédicas que reúnam as seguintes propriedades: baixo módulo de elasticidade, excelente resistência mecânica, assim como boa resistência à corrosão, fácil conformabilidade e sem à presença de elementos tóxicos. Estudos preliminares mostraram que a liga Ti-12Mo- $13 \mathrm{Nb}$ envelhecida a $500^{\circ} \mathrm{C} / 24 \mathrm{~h}$ apresentou alta razão dureza/módulo de elasticidade. Diante do contexto, o objetivo deste trabalho foi analisar o comportamento eletroquímico da liga Ti-12Mo-13Nb envelhecida a $500^{\circ} \mathrm{C} / 24 \mathrm{~h}$, visando seu uso como material biomédico. O comportamento eletroquímico foi realizado por curvas de polarização potenciodinâmica usando solução de $\mathrm{NaCl}$ 0,9 \% para simular o fluido corpóreo. Os resultados mostraram que, a liga Ti-12Mo-13Nb apresentou menores valores de densidade de corrente que a liga Ti-6Al-4V em praticamente toda a faixa estudada.
\end{abstract}

\begin{abstract}
Considerable efforts have been made to explore new Ti alloys for orthopedics applications that combine the following properties: low elastic modulus, excellent mechanical strength, as well as good corrosion resistance, easy adjustment and without the presence of toxic elements. Previous studies had shown that the Ti-12-Mo-13Nb alloy aged $500^{\circ} \mathrm{C} / 24 \mathrm{~h}$ presented high hardness/modulus ratio elasticity. Therefore, the objective of this work was to analyze the electrochemical behavior of the Ti-12-Mo-13Nb alloy aged $500^{\circ} \mathrm{C} / 24 \mathrm{~h}$, aiming at its use as biomedical material. The electrochemical behavior was carried through potentio/dynamic polarization curves using solution of $\mathrm{NaCl} 0.9 \%$ to simulate the body fluid. The results had shown that Ti-12-Mo-13Nb alloy presented lower current density values than the Ti-6Al-4V alloy in, practically, all the levels studied.
\end{abstract}

Key words:

Biomaterials

Titanium Alloys

Corrosion

Microstructure 


\section{Introdução}

Titânio e suas ligas são amplamente usadas como implantes ortopédicos por apresentarem resistência à corrosão, biocompatibilidade, maior resistência e menor módulo de elasticidade que outros biomateriais metálicos tais como aço inoxidável e as ligas a base de Co-Cr ${ }^{[1]}$. A liga de Ti mais utilizada em aplicações ortopédicas é a Ti-6Al-4V. Embora essa liga apresente um menor módulo de elasticidade quando comparada com o aço inoxidável e as ligas de $\mathrm{Co}-\mathrm{Cr}$, esse valor (110-120 GPa) é alto comparado ao do tecido ósseo $(\sim 10-40$ GPa). A diferença entre o módulo do metal e do tecido ósseo pode resultar em reabsorção óssea e eventual falha do implante ${ }^{[2]}$. Além disso, estudos desta particular liga têm mostrado que a liberação de pequenas quantidades dos elementos $\mathrm{V}$ e $\mathrm{Al}$ no corpo humano podem induzir efeitos citotóxicos e desordens neurológicas, respectivamente ${ }^{[3]}$.

Consideráveis esforços têm sido realizados para explorar novas ligas de Ti visando aplicações ortopédicas que reúnam as seguintes propriedades: baixo módulo de elasticidade, excelente resistência mecânica, boa resistência à corrosão, fácil conformabilidade e sem à presença de elementos tóxicos. A fase $\beta$, presente nas ligas de titânio, exibe um menor módulo de elasticidade que as fases $\alpha$ e $\alpha+\beta$, e também, satisfazem a maioria dos pré-requisitos para uma bioliga ideal ${ }^{[4,5]}$ Vários trabalhos na literatura vêm desenvolvendo ligas do tipo $\beta$ compostas de elementos não tóxicos ${ }^{[4,5,6]}$.

Estudos realizados por Gabriel et al. ${ }^{[7,8]}$ sobre o processamento da liga Ti-12Mo-13Nb mostraram que, a maior relação dureza específica, módulo de elasticidade foi obtida na condição envelhecida a $500^{\circ} \mathrm{C} / 24 \mathrm{~h}$. Esse resultado representou um valor da ordem de $37 \%$ em relação à liga Ti-6Al-4V e, portanto, mostrou ter maior potencial para ser aplicada como substituto ósseo, em locais sujeitos a carregamentos mecânicos. Porém, são necessários estudos adicionais como a avaliação da resistência à corrosão desta liga.

Sabe-se que a biocompatibilidade do Ti está intimamente relacionada às propriedades da camada de óxido superficial, em termos de sua estrutura, morfologia e composiç̧̃̃o ${ }^{[9,10]}$. Segundo OKAZAKI GOTOH ${ }^{[11]}$, os metais são liberados dos implantes ortopédicos por vários mecanismos: corrosão, desgaste e processos eletroquímicos mecanicamente acelerados tais como corrosão acelerada por tensão, corrosão por fadiga e corrosão por atrito. Essa liberação tem sido associada com falha do implante, osteólise e reações alérgicas.

Diante do contexto, o objetivo deste trabalho foi analisar o comportamento eletroquímico da liga Ti-12Mo-13Nb obtida sob diferentes condições, visando seu uso como material biomédico.

\section{Procedimento Experimental}

A liga $\mathrm{Ti}-12 \mathrm{Mo}-13 \mathrm{Nb}$ foi preparada a partir de Ti, Mo e $\mathrm{Nb}$ de pureza comercial por fusão a arco com eletrodo não consumível de tungstênio em atmosfera de argônio. $\mathrm{O}$ lingote obtido foi tratado a $1200^{\circ} \mathrm{C}$ por $4 \mathrm{~h}$ em um forno tubular com resfriamento em água a temperatura ambiente e então forjado a frio até redução de $\sim 20 \%$. Em seguida foi tratado a $1000{ }^{\circ} \mathrm{C}$ por $24 \mathrm{~h}$ e resfriamento em água e forjado até redução de $55 \%$. O lingote forjado foi submetido a um tratamento de envelhecimento a $500^{\circ} \mathrm{C} / 24 \mathrm{~h}$ e resfriado em água.

As curvas de polarização potenciodinâmica foram realizadas em potenciostato/galvanostato EG\&G Princeton Applied Research modelo 273-A, tendo como interface o cartão controlador GPIB STD-8410 e aquisição de dados pelo programa Resarch Electrochemistry Software 4.00 (1992) modelo 270 da EG\&G Instruments. Esses testes foram realizados para avaliar o comportamento eletroquímico da liga de Ti-12Mo-13Nb para uso em aplicações biomédicas. As medidas foram realizadas na faixa de potencial entre $-0,4$ e $1,6 \mathrm{~V}$ a uma taxa de varredura de potencial de $0,03 \mathrm{~V} \cdot \mathrm{min}^{-1}$. Os ensaios foram realizados a $250 \mathrm{C}$ usando uma célula de vidro com tampa de teflon contendo $15 \mathrm{~mL}$ de solução de $0,9 \%$ de $\mathrm{NaCl}$. Ligas de Ti-12Mo-13Nb e Ti-6Al-4V, ambas com área de $0,17 \mathrm{~cm}^{2}$, foram usadas como eletrodo de trabalho. Fio de platina em espiral foi utilizado como contraeletrodo e prata/cloreto de prata $(\mathrm{Eo}=0.222 \mathrm{~V})$, em solução de 1 mol.L-1 de KCl, como eletrodo de referência. As curvas potenciodinâmica foram realizadas em duplicata e antes de cada teste, as ligas foram polidas com alumina e ácido oxálico, rinsadas com água destilada e álcool, e secas. 


\section{Resultados e Discussão}

A Figura 1 apresenta duas imagens de campo claro obtidas por microscopia eletrônica de transmissão da liga envelhecida a $500{ }^{\circ} \mathrm{C} / 24$ h. A primeira imagem (a) mostra lamelas da fase $\alpha$ na matriz $\beta$ e a segunda (b) mostra a fase $\alpha$ na forma de partículas não lamelares na matriz $\beta$. A Figura 1 (c) mostra o padrão de difração da liga onde se identifica as fases $\alpha$ e $\beta$, respectivamente. Portanto, com o envelhecimento a microestrutura desta liga é composta somente por fase $\alpha$ na matriz $\beta^{[7,8]}$.

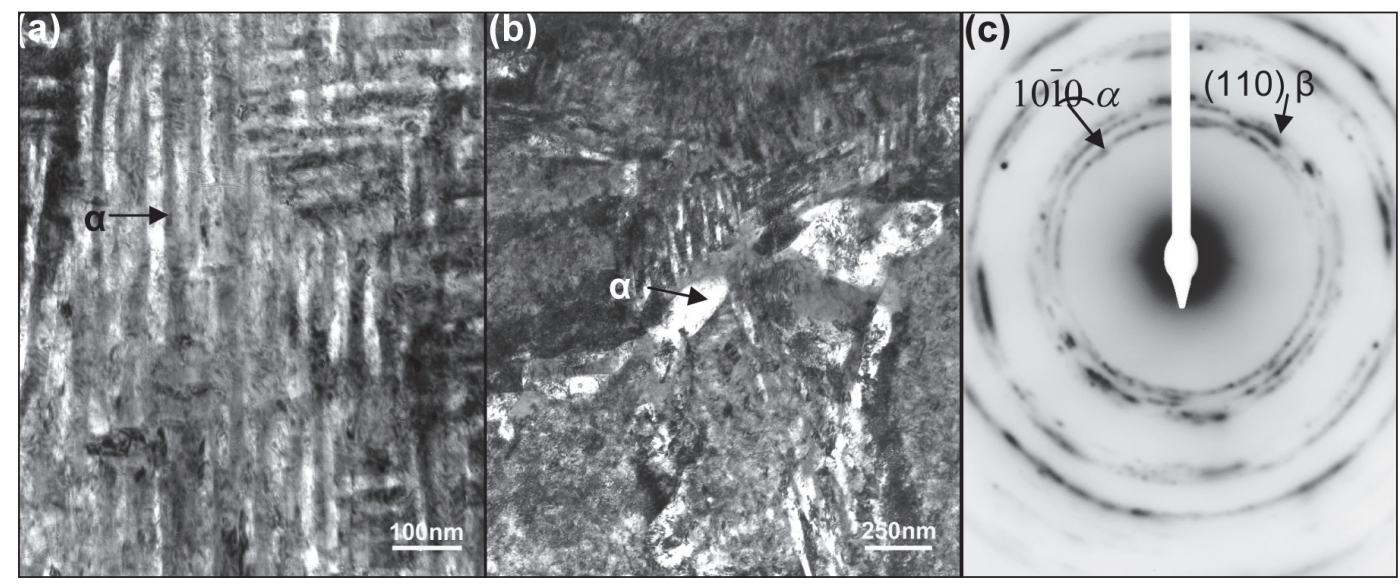

Figura 1 - Análises por MET da liga Ti-12Mo-13Nb envelhecida a $500{ }^{\circ} \mathrm{C} / 24 \mathrm{~h}$ : (a) imagem de campo claro de lamelas da fase $\alpha$ (branco) em uma matriz $\beta$ (b) imagem de campo claro de partículas não lamelares da fase $\alpha$ (branco) em uma matriz $\beta$ e (c) padrão de difração com indicação das fases $\alpha$ e $\beta^{[7,8]}$.

Em relação às propriedades mecânicas da liga nesta condição, o módulo de elasticidade e a dureza da liga foi de aproximadamente $84 \mathrm{GPa}$ e $320 \mathrm{HV}$, respectivamente.

Para medir a desempenho de biomateriais com baixo módulo de elasticidade e alta resistência para serem utilizados como substituto ósseo, geralmente utiliza-se a razão resistência por módulo de elasticidade, onde quanto maior o valor resultante, maior será o potencial para uso nestas aplicações. Assim sendo, a razão dureza específica por módulo da liga nesta condição representou um valor da ordem de $37 \%$ em relação à liga comercial Ti-6Al-4V [7,8].

As curvas de polarização potenciodinâmica das ligas Ti-12Mo-13Nb envelhecida a $500{ }^{\circ} \mathrm{C} /$ 24 h e Ti-6Al-4V em solução de $0,9 \%$ de $\mathrm{NaCl}$, simulando a agressividade do corpo humano, foram realizadas para avaliar a resistência à corrosão da liga Ti-12Mo-13Nb, conforme mostra a Figura 2. A liga Ti-6Al-4V foi utilizada para efeito de comparação, por se tratar de um material comumente usado em implantes ortopédicos ${ }^{[6]}$.

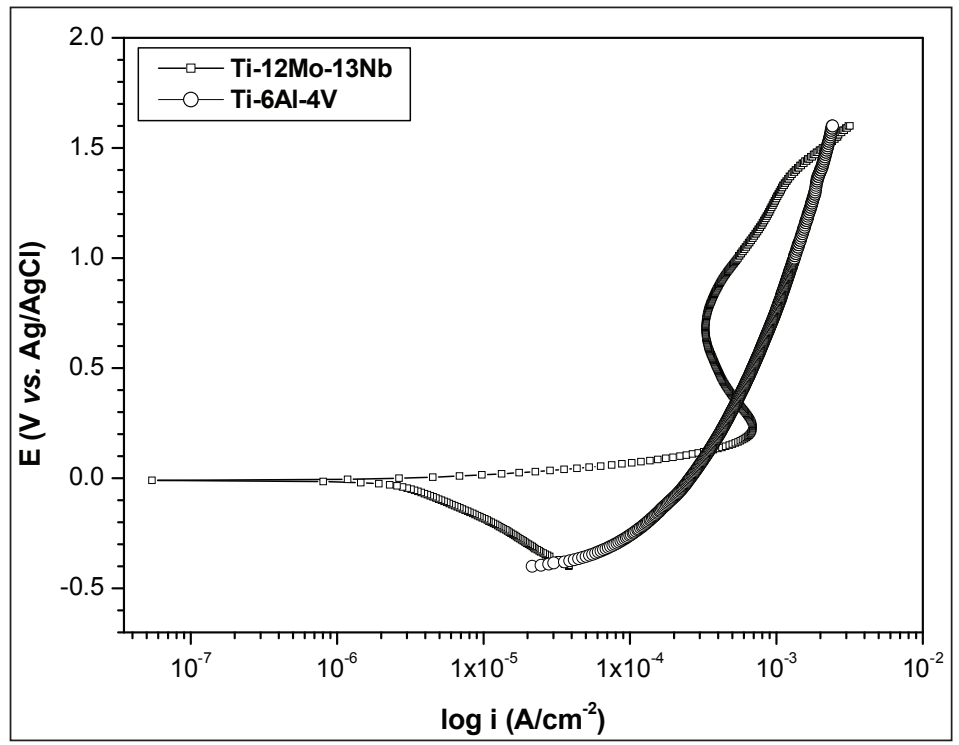

Figura 2- Curvas de polarização potenciodinâmica das ligas de Ti-12Mo-13Nb e Ti-6Al-4V em solução de $0.9 \%$ de $\mathrm{NaCl}$ a uma taxa de varredura de potencial de $0,03 \mathrm{~V} \cdot \mathrm{min}^{-1} \mathrm{e}$ a $25 \mathrm{oC}$. 
A região anódica da curva de polarização da liga Ti-12Mo-13Nb entre 0,2 e 1,5V indica a formação de uma região passiva, associada à formação de um ou mais filme protetor ${ }^{[12]}$. Não foi observada região de formação de pite e nem oscilação da densidade de corrente na região passiva, indicativo da presença de corrosão por pite ${ }^{[12]}$. Observa-se ainda que, ocorreu uma estabilização parcial da densidade de corrente em 8x10-7 A.cm ${ }^{-2}$, indicando que o filme de óxido de titânio foi formado ${ }^{[13]}$. Entretanto, esse filme torna-se mais estável em potenciais superiores a $0,16 \mathrm{~V}$ onde se observa uma leve estabilização da densidade de corrente com o aumento do potencial. Através da curva potenciodinâmica da liga Ti-12Mo$13 \mathrm{Nb}$, foram obtidos os seguintes resultados: Ecorr. $=-15,717 \mathrm{mV}$, Icorr. $=0,0544 \mu \mathrm{m} . \mathrm{cm}^{-2}$ e Ipass. $=622 \mu \mathrm{m} . \mathrm{cm}^{-2}$. Os resultados mostraram ainda que, a liga Ti-12Mo-13Nb apresentou menores valores de densidade de corrente que a liga Ti-6Al-4V em praticamente toda a faixa estudada.

\section{Conclusão}

Conclui-se que a liga Ti-12Mo-13Nb na condição envelhecida com uma microestrutura $\alpha+\beta$ apresentou além de baixo módulo de elasticidade e alta dureza em relação a liga comercial Ti-6Al-4V, uma boa resistência à corrosão. Quando comparada com a liga comercial Ti-6Al-4V, apresentou menores valores de densidade de corrente que esta em praticamente toda a faixa estudada. Portanto, esta avaliar a resistência à corrosão da liga em função do tempo de exposição em solução de $0,9 \%$ de $\mathrm{NaCl}$ liga é um promissor candidato para ser utilizado como implante, porém, serão necessários estudos adicionais envolvendo outras análises como: testes de fadiga, citotoxicidade, teste in vivo e medidas de potencial de circuito aberto (OCP) para melhor.

\section{Agradecimento}

Os autores agradecem ao Departamento de Engenharia de Materiais da Escola de Engenharia de Lorena (EEL/USP) pelo desenvolvimento deste trabalho.

\section{Referências}

1. ZHOU, Y. L., NIINOMI, M., AKAHORI, $\mathrm{T}$, et al., Corrosion resistance and biocompatibility of Ti-Ta alloys for biomedical applications, Materials Science and Engineering A, v. 398, p. 28-36, 2005.

2. MAJUMDAR, P., SINGH, S. B., CHAKRABORTY, M., Elastic modulus of biomedical titanium alloys by nanoindentation and ultrasonic techniques - A comparative study, Materials Science and Engineering A, v. 489, p. 419-425, 2008.

3. RAABE, D., SANDER, B., FRIÁK, M., et al., Theory-guided botton-up design of $\beta$-titanium alloys as biomaterials based on first principles calculations: Theory and experiments, Acta Materialia, v. 55, p. 4475-4487, 2007.

4. LI, S. J., YANG, R., LI. S., et al., Wear characteristics of Ti-Nb-Ta-Zr and Ti6Al-4V alloys for biomedical applications, Wear, v. 257, p. 869-876, 2004.

5. XU, W., KIM, K.B., DAS, J., et al., Phase stability its effect on the deformation behavior of Ti-Nb-Ta-In/Cr $\beta$ alloys, Scripta Materialia, v. 54, p. 1943-1948, 2006.

6. GORDIN, D.M. et al. Synthesis, structure and electrochemical behavior of a beta Ti-12Mo-5Ta alloy as new biomaterial, Materials Letters, v. 59, p.2959-2964, 2005.

7. GABRIEL, Sinara Borborema. Processamento e caracterização de ligas Ti-Mo-Nb para aplicações biomédicas, 2008, Tese de Doutorado, COPPE/UFRJ, Rio de Janeiro, 2008.

8. GABRIEL, S. B., NUNES C. A., DILlE, J., SOARES, G. A. Microstructural evolution and Mechanical properties of aged Ti-12Mo-13Nb alloy. In Congresso Brasileiro de Engenharia e Ciências dos Materiais, Porto de Galinhas, 2008.

9. LI, L., KONG, Y., KIM, H., et al., Improved biological performance of $\mathrm{Ti}$ implants due to surface modification by micro-arc oxidation", Biomaterials, v. 25, p. 2867-2875, 2004. 
10. NIINOMI,M.,AKAHORI, T., TAKEUCHI,

T., et al., Mechanical properties and cytotoxicity of new beta type titanium alloy with low melting points for dental applications, Materials Science and Engineering C, v. 25, p. 417-425, 2005.

11. OKAZAKI, Y., GOTOH, E., Comparison of metal release from various metallic biomaterials in vitro, Biomaterials, v. 26, p. 11-21, 2005.

12. AlveS, A. P. R., SANTANA, F. A., ROSA, L.A. A., et al., A study on corrosion resistance of the Ti-10Mo experimental alloy after different processing methods, Materials Scienc and Engineering, v. C24, p. 693-696, 2004.

13. De Assis, S. L., Wolynec, S., Da Costa, I., Corrosion characterization of titanium alloys by Electrochemical techniques, Electrochimica Acta, v.51, p. 1815-1819, 2006.

Endereço para Correspondência:

Sinara Borborema Gabriel

sinarab@msn.com

Centro Universitário de Volta Redonda

Campus Três Poços

Av. Paulo Erlei Alves Abrantes, $n^{\circ} 1325$,

Três Poços - Volta Redonda / RJ

CEP: 27240-560

Informações bibliográficas:

Conforme a NBR 6023:2002 da Associação Brasileira de Normas Técnicas (ABNT), este texto científico publicado em periódico eletrônico deve ser citado da seguinte forma: SILVA, Tales Vieira, SANTOS, Iranildes dos; GABRIEL, Sinara Borborema. Comportamento Eletroquímico da Liga Ti-12mo-13nb envelhecida para aplicação Biomédica. Cadernos UniFOA. Volta Redonda, Ano V, n. 14, dezembro 2010. Disponível em: <http://www.unifoa.edu.br/cadernos/edicao/14/19.pdf> 